

\title{
8 Representaciones sociales sobre el rol del docente tutor en modalidades b-learning de la Universidad Militar Nueva Granada
}

\author{
José Eduardo Padilla Beltrán \\ Diego Armando Rincón Caballero \\ Laura Jimena Buitrago Duarte
}

\section{Introducción}

$\mathrm{D}$ orfsman (2012) explica que los cambios en la profesión docente son parte de las exigencias de la sociedad de la información según la gestión del quehacer a través de dimensiones disciplinares, pedagógicas, personales y comunitarias que tienen repercusiones en lo académico y personal de los docentes. Este autor afirma que las dimensiones del docente del siglo pasado en cuanto a su acción liberal, normalizadora y profesional se han visto modificadas por las transformaciones tecnológicas, sociales y políticas que generan en estos actores incertidumbre por el crecimiento de las tecnologías de la información y de la comunicación (TIC) en el campo educativo y la percepción frente a estas mediaciones tecnológicas en los diferentes sistemas educativos por reducir brechas de desigualdad, acceso al conocimiento y potencial en la comunicación.

Por tanto, el argumento central de este capítulo es establecer un nivel de relación e interdependencia entre los roles que asume el docente en la modalidad b-learning frente a determinadas condiciones enmarcadas en lo institucional y político del contexto en el cual se realiza el quehacer profesional. Por ello, analizar e investigar sobre estos roles permite entender la necesidad de nuevos espacios de formación, apropiación y evaluación de docentes en esta modalidad educativa.

\footnotetext{
José Eduardo Padilla Beltrán. Licenciado en Ciencias de la Educación por la Universidad Pedagógica Nacional, magíster en Administración y Supervisión Educativa por la Universidad Externado de Colombia, magíster en Evaluación en Educación por la Universidad Santo Tomás, Doctor of Education por la Newport University y Posdoctor en Educación, Ciencias Sociales e Interculturalidad por la Universidad Santo Tomás. Director del Centro de Investigaciones de la Facultad de Estudios a Distancia. e-mail: eduardo.padilla@unimilitar.edu.co
}

Diego Armando Rincón Caballero. Licenciado en Diseño Tecnológico y magíster en Educación por la Universidad Pedagógica Nacional. Asistente de Investigación del Grupo PYDES en la Universidad Militar Nueva Granada. e-mail: dccaballero24@gmail.com

Laura Jimena Buitrago Duarte. Profesional en Relaciones Internacionales y Estudios Políticos por la Universidad Militar Nueva Granada y maestranda en Relaciones y Negocios Internacionales en la misma universidad. Joven Investigadora del Grupo PYDES en la Universidad Militar Nueva Granada. e-mail: jimenabuitrago.d@gmail.com 
En la Recomendación conjunta de la OIT y la Unesco relativa a la situación del personal docente (1966) y Recomendación de la Unesco relativa a la condición del personal docente de enseñanza superior (1997) (Unesco, 2008), se establece un aporte respecto de normativa que tiene como epicentro el sentido profesional, social, ético y material de los docentes. En cada uno de estos ejes, se conciben temas relacionados con la formación inicial, la estabilidad en la profesión, los asensos, los procesos disciplinarios, la libertad de cátedra, los derechos y las responsabilidades, así como la posibilidad de brindar condiciones para que el ejercicio pedagógico se realice de manera idónea. Sin embargo, como aclara la OCDE (2009), cada vez más la elección de las carreras de docencia es menos interesante para las personas que aspiran a estudiar en la universidad, por tanto, este organismo plantea la problemática de atraer buenos docentes, y ampliar procesos y políticas educativas para captar talentos y personas interesadas en la educación.

En términos de la teoría del rol social de Eagly (1987), si los papeles son asignados en función única del trabajo, la primera consecuencia es la estereotipación que repercute en desigualdades, conflicto de identidad y pérdida de interés en la labor docente. En otra perspectiva de la acción social, está el postulado de Erving Goffman enunciado por Herrera y Soriano (2004), caracterizado más por la evocación dramatúrgica, en la cual el rol es la acción de intentar representar un personaje que incluye "los conceptos de complejo de roles (roleset), sectores de rol (o subroles), funciones de rol (funcionales/disfuncionales, manifiestas/latentes), compromiso de rol, conflicto de rol y disenso de rol (interrol e intrarrol), roles ocultos, 'jugar a un rol' (p. 65).

Esta situación se torna compleja si se analiza el rol del docente universitario en espacios no presenciales, por lo cual Cebrián de la Serna y Vain (2008) plantean que el modelo dominante cambia y el escenario o epicentro de la clase se modifica al integrar otros espacios dentro y fuera de la instituciones de educación superior para la interacción con el conocimiento y los educandos. Por esta razón, la dificultad más prominente en la asunción del rol en esta perspectiva social es la preocupación del docente por las prácticas de enseñanza y la representación del estudiante, es decir, el desconocimiento de esa persona que se considera está alejada en un marco espacio-temporal, lo cual hace difícil el supuesto de interacción cara a cara de Goffman en lo psicosocial del rol (Herrera y Soriano, 2004).

En América Latina, Salinas (2008) afirma que el rol principal del docente estará esbozado en la habilidad de diseñar situaciones, medios y oportunidades para que el estudiante se acerque al conocimiento. No obstante, para Morresi y Donnini (2007, p. 4), el rol principal del docente es el de tutor, conceptualizado como "un agente que guía, orienta y evalúa el aprendizaje, brindando la mejor metodología de enseñanza dirigida al alumno". Esta percepción, en contraste, permite exponer la afirmación de Méndez, Rivas y Del Toro (2007), quien 
afirma que el tutor es un "Docente. Profesional que imparte el programa, de acuerdo con los materiales previamente elaborados, y que interactúa directamente con el estudiante o participante" (p. 35).

Hay otras posturas que comprenden el rol desde el conjunto de funciones y desempeños, toda vez para Cataldi, Figueroa, Lage, Kraus, Britos y García (2005) el perfil es un componente fundamental de caracterización del rol docente respecto de lo disciplinario y la interacción comprometida con el aprendizaje del estudiante. Además, Zambrano, Medina y García (2010) refuerzan esta óptica del perfil docente, tratando de reflexionar en los componentes humanísticos, centrados en la ética coherente con la pedagogía y didáctica por desarrollar en la educación superior. Asimismo, Dellepiane y Klas (2010, p. 3) sugieren del perfil docente el "modo de ser" del cual se desprende toda una serie de compromisos con la institución; de indole profesional, reflejados en el sentido de pertenencia; y en el desempeño, promoviendo estrategias pedagógicas innovadoras en el aula.

Para Vaillant (2005), las reformas educativas en el siglo XXI han propuesto el retorno al protagonismo y la centralidad de la labor docente que esté direccionada por áreas, tales como la calidad, el currículo, el perfeccionamiento docente, la gestión y la evaluación. No obstante, como la autora explica, este proceso ha sido tardío e inconcluso, presentando políticas parciales relativas al docente en América Latina, en cuanto muestran la carrera docente caracterizada específicamente por los cargos, las funciones y los asensos a los cuales se aspira.

En razón de esto, el rol atribuido a los docentes para dar respuesta a los cambios en la educación y la sociedad del siglo XXI es la de "orientador, facilitador y gerente del proceso educativo" (Colbert y Castro, 2014, p. 10), toda vez que se modifican concepciones de espacio y tiempo inherentes al paso de transmitir información por parte del docente a ser el creador de ambientes de aprendizaje necesarios para la educación crítica y creativa. De esta perspectiva aún se emplea el concepto de rol respecto del desarrollo profesional y la limitación del deber ser acorde con la calidad educativa más que a la educación de calidad.

De lo anterior, la teoría del rol ampliada al referente social expone que este no es la conducta ni la función misma ejecutada por el sujeto; por tanto, el rol está más vinculado a la situación de interacción, en cuanto puede asumir diferentes roles dependiendo de los demás sujetos, el espacio o la comunicación efectuada a partir de convenciones sociales. Sarbin (1968, p. 93) emplea el concepto de self como el grado de asociación entre la persona y el papel actuado en las diferentes interacciones, lo cual trae consigo el juego de roles que pueden llevarlo a ser líder o seguidor en el proceso educativo. 
La introducción de los aspectos psicosociales de la teoría del rol en el campo de la praxis del docente tiene repercusiones que pueden dar fundamento a la brecha o proximidad de las expectativas sociales y la personalidad de los docentes en cuanto la apropiación del rol en determinado contexto (Loscertales, 1989). En esa lógica psicosocial, los problemas del rol se acentúan, no en la cantidad y calidad de las funciones por realizar, sino en el balance del self y el personaje recreado en el ámbito profesional, que dan paso a los conflictos de comunicación, inadaptación y trasposición con otros roles más personales que pueden generar disonancia en el momento de interactuar con otros actores educativos (Loscertales, 1989).

En la educación superior nacional, Arboleda y Rama (2013) señalan que uno de los grandes desafíos en la modalidad b-learning es lograr que el docente se convierta en facilitador de conocimientos, mediando entre estudiantes, tecnologías, contenidos y situaciones problemáticas tendientes al conocimiento significante. Por tanto, su rol está supeditado a la capacidad y voluntad de perfeccionar la labor, tanto en el campo disciplinar como en lo pedagógico, en acopio a procesos de investigación que permitan fundamentar nuevas prácticas educativas con mayor énfasis en la motivación y la orientación.

En lo político, el Ministerio de Educación Nacional (2009) plantea que la virtualidad está en plena construcción, teniendo como disyuntivas lo efectuado desde aquel por medio de sus dependencias para la educación virtual y a distancia, y lo propuesto respecto de las TIC por el Ministerio de Tecnologías de la Información y la Comunicación, centrado en la parte infraestructural y civil del uso de estas tecnologías. En esa medida, se puede pensar que la formación del sujeto entra en separaciones de una educación formal y otra del ciudadano alfabetizado en las TIC, lo cual trae consigo en la docencia el problema de desconocimiento del panorama político que incide en la comunidad educativa.

En ese marco, se plantean roles como el de facilitador, consejero, experto en las TIC, verificador de evidencias y líderes constructores (Sabogal, 2014), cada uno con tareas que deben desarrollarse para el alcance de los objetivos de aprendizaje. En razón de esto, en el ámbito nacional, la propuesta del rol ha sido abarcada desde la concepción de las funciones, los perfiles y los desempeños que el docente debe llevar a cabo en las modalidades b-learning, en cuanto estas acciones permiten evaluar y gestionar el quehacer de acuerdo con parámetros institucionales y contextuales en los cuales desarrolla su profesión.

Algunos estudios propuestos en la Universidad Militar Nueva Granada, como los de Padilla, Páez y Montoya (2008) proponen las categorías de rol, identidad, actitud y creencias para interpretar la forma en que los docentes usan de manera cotidiana y en su quehacer profesional las TIC, concluyendo que muchos docentes no diferencian entre 
los roles que se pueden asumir de manera presencial y virtual y la adquisición de "una formación empírica, obtenida por experiencia del día a día dentro de los procesos de enseñanza" (p. 56).

Por otro lado, González, Padilla y Rincón (2012) se proponen algunos roles en relación con etiquetas que ayuden a catalogar las funciones que cumple el docente en esta modalidad, cuyo fin es el de evaluar el desempeño profesional. Dentro de estos roles, se encuentran los de diseñador, facilitador de aprendizaje, comunicador, tutor y administrador como pilares en la interacción, seguimiento y realimentación de los educandos y pares profesionales que intervienen en la construcción y el desarrollo de asignaturas propuestas en esta modalidad semipresencial.

En razón de lo expuesto, la formulación del problema se declara en ¿qué representaciones sociales tienen los docentes de la Facultad de Estudios a Distancia de la Universidad Militar Nueva Granada sobre el rol profesional en la modalidad b-learning de aprendizaje? De este modo, la interpretación del aspecto representacional está supeditada al enfoque psicosocial del rol a manera de autorrepresentación del quehacer docente.

\section{Método}

Strauss y Corbin (2002) plantean dentro del método comparativo constante el concepto de muestreo teórico, cuyo fundamento es la selección de datos por ser comparados y contrastados de acuerdo con el campo teórico que se investiga; en este caso, lo concerniente al marco representacional del rol docente. Igualmente, dentro de este acervo metódico, se incluye el concepto de saturación, el cual no tiene pretensión de generalidad, en cuanto la razón de validez está en la recolección de información consecuente al proceso de zigzag. Este último consiste en ir al campo a recolectar los datos en varias etapas y, posteriormente, redundar en nuevas interpretaciones en busca de descriptores que permitan organizar la información, lo cual se efectúa a través del software ATLAS.ti. ${ }^{18}$

De lo anterior, el método comparativo constante articula las modalidades de obtención de datos, codificación e interpretación durante todo el proceso de ida y vuelta al campo de saturación de datos que implica el componente holístico acorde con el enfoque cualitativo (Sautu, Boniolo, Dalle y Elbert, 2005; Valles, 2003). En razón de esto, el uso de ATLAS.ti permite llevar el sucesivo de los datos interpretados y la descripción a través de memorandos

18 Se utiliza la versión licenciada 6.1.28 adqurida por la Universidad Militar Nueva Granada bajo el tipo de licencia académica. 
que dan cuenta de los datos recopilados y los significados producto de operaciones de codificación (Strauss y Corbin, 2002).

\section{Población del estudio}

Esta se define a través de un estudio de caso como el conjunto de docentes adscritos a la Facultad de Estudios a Distancia de la Universidad Militar Nueva Granada que poseen experiencia en modalidades $b$-learning con vinculación de tiempo completo y medio tiempo en los programas de pregrado: Administración de Empresas (5 sujetos), Contaduría Pública (4 sujetos), Relaciones Internacionales y Estudios Políticos (3 sujetos), Ingeniería Civil (5 sujetos), Ingeniería Industrial (2), Administración de la Seguridad y Salud Ocupacional (4 sujetos), y de la misma manera docentes de posgrado en el programa de Especialización en Alta Gerencia (4 sujetos). Por consiguiente, la muestra se estima en 27 docentes con estos atributos, que representa $35 \%$ del universo de docentes adscritos a la Facultad de Estudios a Distancia en diferentes programas y vinculaciones (77).

\section{Técnicas e instrumentos de recolección de datos La asociación libre}

Esta técnica es indispensable para acceder al universo semántico sin tener la caracterizada expresión discursiva por parte del informante; por consiguiente, se recurre a un término inductor (Abric, 2001) que permite desencadenar una serie de producciones verbales ligadas al conjunto de creencias, pensamientos y supuestos del objeto de estudio. En esa instancia, el informante recurrió a términos, adjetivos y apelativos que estuvieron en su mente, pero se inscriben en el conjunto de interacciones e intercambios de orden sociocultural para proyectar los pensamientos sobre dicho objeto social (rol docente). Al mismo tiempo, esta técnica requiere menos control que las interrogativas, en cuanto se delega un nivel más espontáneo al sujeto y se persigue el acceso más rápido a la representación.

\section{Los tris jerarquizados}

Dependiente de la asociación libre inicial, los tris jerarquizados recopilan los elementos de la asociación y establecen a través del ítem de frecuencia los aspectos más relevantes (Abric, 2001), más o menos 32 términos. Además, se solicita al sujeto dividir las fichas de cada elemento entre las más acordes con el objeto y las que menos; por consiguiente, repite la misma operación al formar paquetes de 8,4 y 2 en cada criterio de relación con el objeto de la representación, lo cual permite el análisis del rango medio de los términos utilizados en 
Comunicación, redes, aprendizaje y desarrollo institucional y social

la caracterización del último paquete y así identificar los elementos centrales junto con la asociación libre que otorga la frecuencia de los términos desde el enfoque estructural. Estos tris jerarquizados se organizan en el programa ATLAS.ti según las co-ocurrencias, por ende, permite dar paso a la codificación de los elementos a través de los criterios de fundamentación y densidad dispuestos por el software.

\section{Análisis de la información}

La teoría fundamentada propende a la flexibilidad inductiva capaz de integrar aspectos desconocidos o poco tratados del objeto de estudio. Según esta cualidad, la teoría fundamentada incluye elementos por contrastar de forma permanente con suposiciones que vayan surgiendo a causa de la adición de nuevos datos en el estudio. Al respecto, la primera etapa que considera esta propuesta metodológica tiene que ver con el uso de códigos provisionales para clasificar y operar datos recolectados que logran la fragmentación analítica consecuente al momento temporal de la familiarización del investigador con los elementos emergentes del objeto de estudio (rol docente) (Strauss y Corbin, 2002). A este proceso se le denomina codificación abierta y funciona como primera medida de descripción sobre la abstracción de los datos recolectados.

Luego de la codificación abierta, Araya (2002) sugiere la instancia de "reconstrucción del núcleo figurativo" (p. 72), en cuanto se llevan a cabo dos procesos: el primero inherente a la codificación axial que busca conexiones entre los conceptos y las categorías establecidas durante la codificación abierta a partir de las dimensiones e incidencias de cada una en el contenido representacional; la segunda centrada en la codificación selectiva a través de la relación de los contenidos descriptivos hasta dotarlos contextualmente en orden argumentativo y, por último, a modo de esquema teórico, se propicia la identificación de categorías principales por medio de la discriminación de ocurrencias y densidad entre los códigos operativos ofrecidos por el software ATLAS.ti.

\section{Resultados}

Se sistematizaron los datos adquiridos en las producciones semánticas de la muestra poblacional, aplicando en cada término inductor los criterios de frecuencia, rango y palabra clave (Abric, 2001) en la producción que se desarrolló (cinco palabras, adjetivos o composiciones relacionadas con la palabra estímulo). En razón de esto, las palabas estímulo derivan de los antecedentes del estudio. A continuación, se presentan los términos inductores utilizados con el respectivo análisis de frecuencia rango e importancia. 
Para el término inductor de b-learning, el rango de repeticiones de las cadenas se encuentra en nueve, toda vez que la palabra más importante señalada por la muestra poblacional es aprendizaje. De este modo, la frecuencia en las palabras enunciadas por los docentes se enfoca en la repetición del aprendizaje (figura 8.1).

Figura 8.1. Distribución de frecuencia del término b-learning.

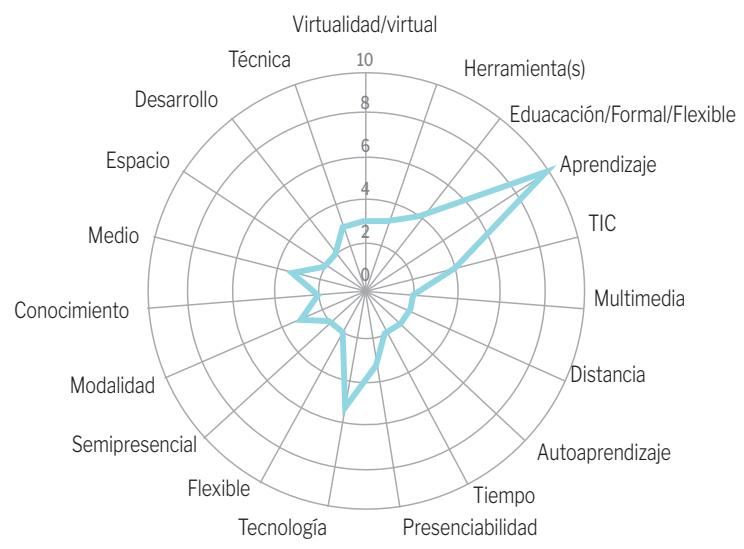

Fuente: Elaboración propia.

En el caso del término inductor de docencia, la palabra que adquirió mayor importancia es la de enseñanza, en cuanto simultáneamente es la palabra con más repeticiones en la distribución de frecuencia. Asimismo, el rango de repetición se encuentra orientado en el orden de las siete palabras, por ende, se evidencia una dispersión de términos en las cadenas semánticas.

La palabra con mayor frecuencia y a la vez de mayor importancia en el término TIC es la tecnología. Por otro lado, el rango de aparición es de once, por lo cual se halla dispersión en el conjunto de palabras usadas para describir las TIC (figura 8.2). 
Comunicación, redes, aprendizaje y desarrollo institucional y social

Figura 8.2. Distribución de frecuencia del término TIC.

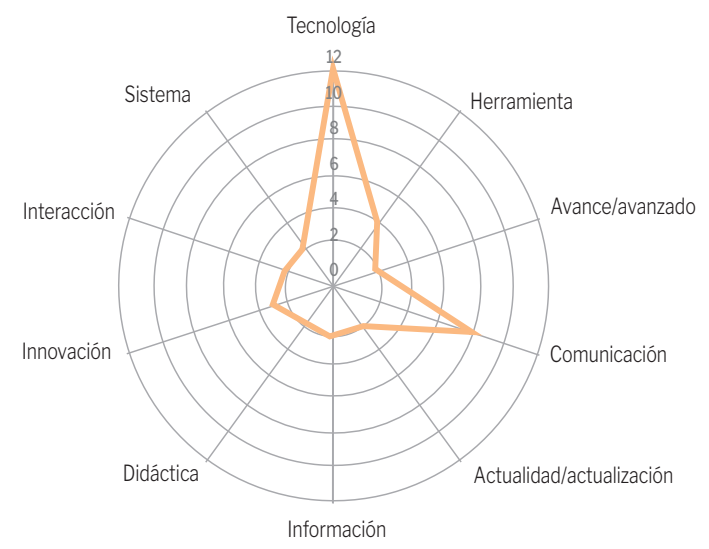

Fuente: Elaboración propia.

En el término inductor de pedagogía, la frecuencia se centra en la palabra enseñanza, no obstante, elementos como el aprendizaje adquieren relevancia en la producción semántica designada por los docentes. Consecuentemente, el rango de aparición es de diez, teniendo un contraste entre la palabra con mayor repetición (enseñanza) y otras con baja aparición como en el caso de arte, educación y didáctica (figura 8.3).

Figura 8.3. Distribución de frecuencia del término pedagogía.

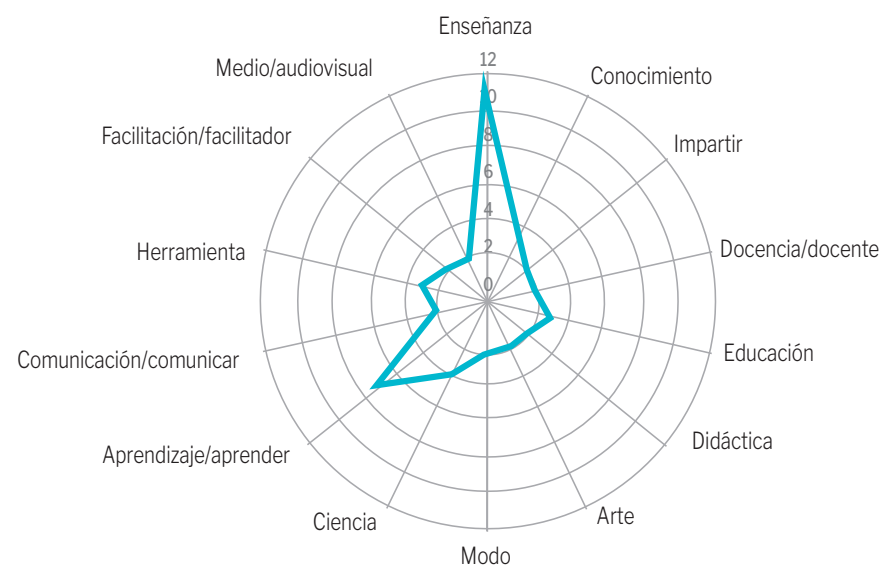

Fuente: Elaboración propia. 
Para el término inductor rol, la frecuencia se centra en el vocablo papel. Asimismo, el rango de repetición es de nueve palabras que adquirieron un relativo consenso ante los anteriores términos inductores.

Figura 8.4. Distribución de frecuencia del término rol.

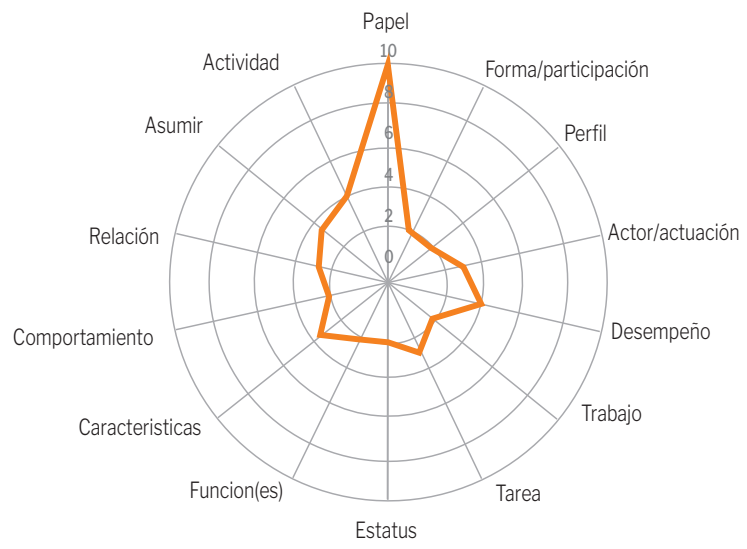

Fuente: Elaboración propia.

En particular, para el término inductor facilitador, hay un rango de aparición de cinco palabras, por ende, se encuentran múltiples léxicos en los cuales existen acuerdos (figura 8.5). En razón de esto, la frecuencia adquiere distribución uniforme, aunque sea preponderante para la palabra orientador. Sin embargo, las palabras señaladas como las más importantes en la cadena semántica son ayuda y acompañamiento.

Figura 8.5. Distribución de frecuencia del término facilitador.

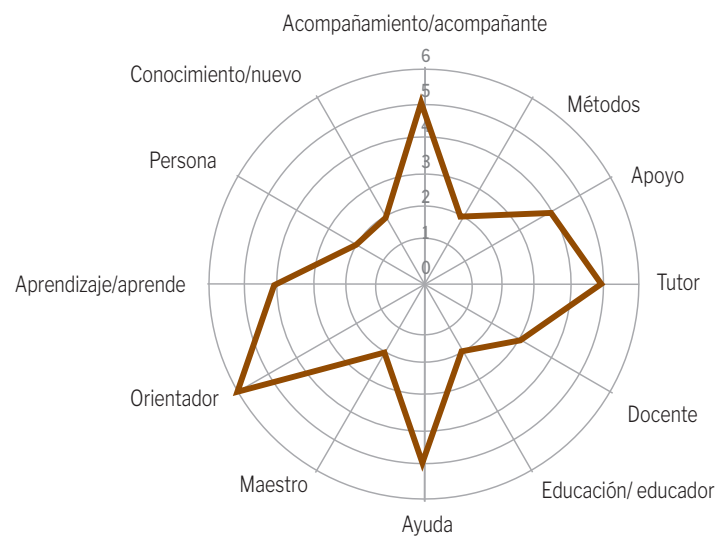

Fuente: Elaboración propia. 
En contraste con lo anterior, el rango de repetición para el término inductor de orientador es de once palabras que adquiere mayor frecuencia en la palabra guía, la cual es señalada como importante seguida del elemento de facilitador.

La distribución de frecuencia de la dicción tutor se concentró en la palabra guía, la cual también es reseñada como las más importantes junto a la expresión de orientador. De otro modo, el rango de aparición es de diez palabras, y adquieren menor aparición palabras como profesor o docente (figura 8.6).

Figura 8.6. Distribución de frecuencia del término tutor.

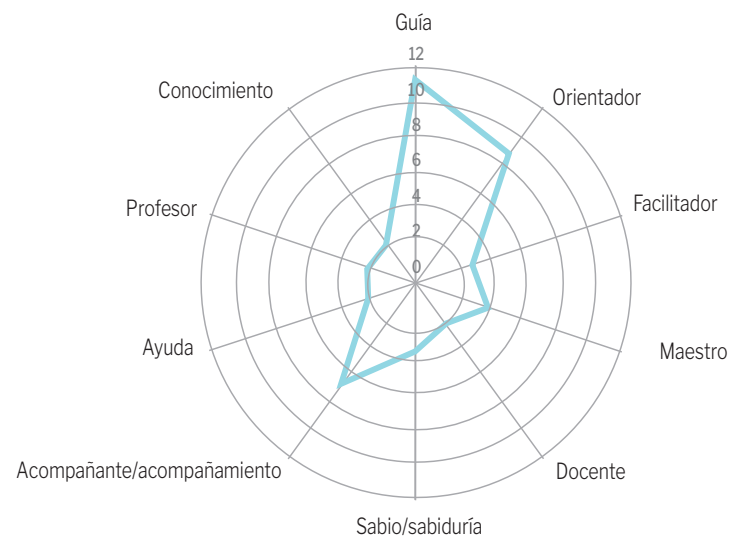

Fuente: Elaboración propia.

El perfil docente como término inductor se equipara en el ámbito de frecuencia en las características, al igual que es la palabra más importante en las producciones semánticas. De lo anterior, el rango de aparición de ocho palabras refleja el grado de dispersión considerable en otras palabras con pocas iteraciones.

El rango de repetición del término inductor investigador es de tres que presenta exiguos picos de frecuencia, como en el caso del término facilitador, por tanto, la frecuencia se concentra en la palabra conocimiento seguida de elementos como buscar o curiosidad. Acorde con esta situación, no se encontró palabra importante en la cadena de palabras.

En el término trabajo flexible, se emplea el mismo estímulo para describir el término inductor, por lo que la palabra trabajo se encuentra frecuentemente acompañada por otros adjetivos. Igualmente, la expresión trabajo es manifestada como la más importante por la muestra, que incluye el rango de aparición de siete palabras (figura 8.7). 
Figura 8.7. Distribución de frecuencia del término trabajo flexible.

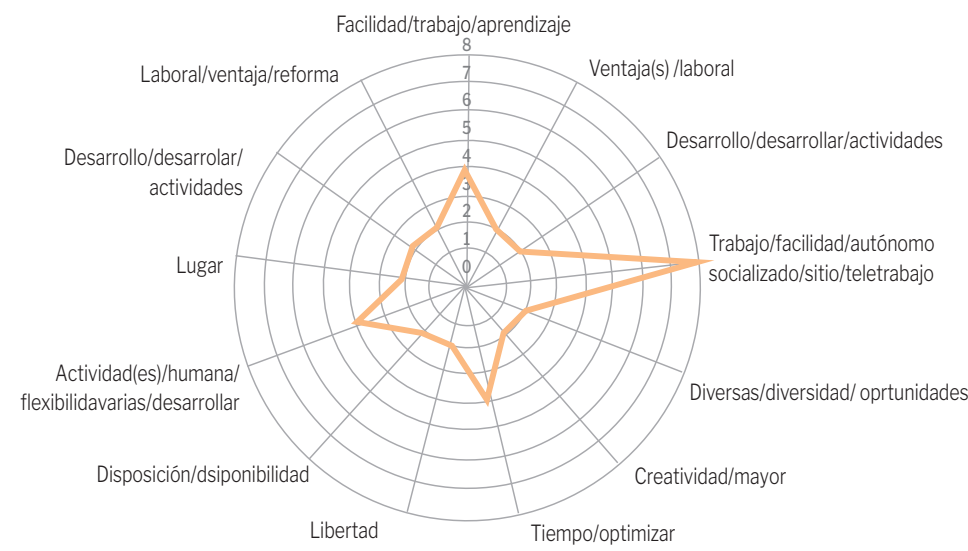

Fuente: Elaboración propia.

El término inductor de proceso cognitivo evoca en la frecuencia la palabra aprendizaje (figura 8.8), la cual es acompañada por otros elementos, tales como el proceso, esta última señalada, además, como la dicción más importante por la muestra poblacional. En cuanto al rango de repetición, se tiene un valor de seis palabras.

Figura 8.8. Distribución de frecuencia del término proceso cognitivo.

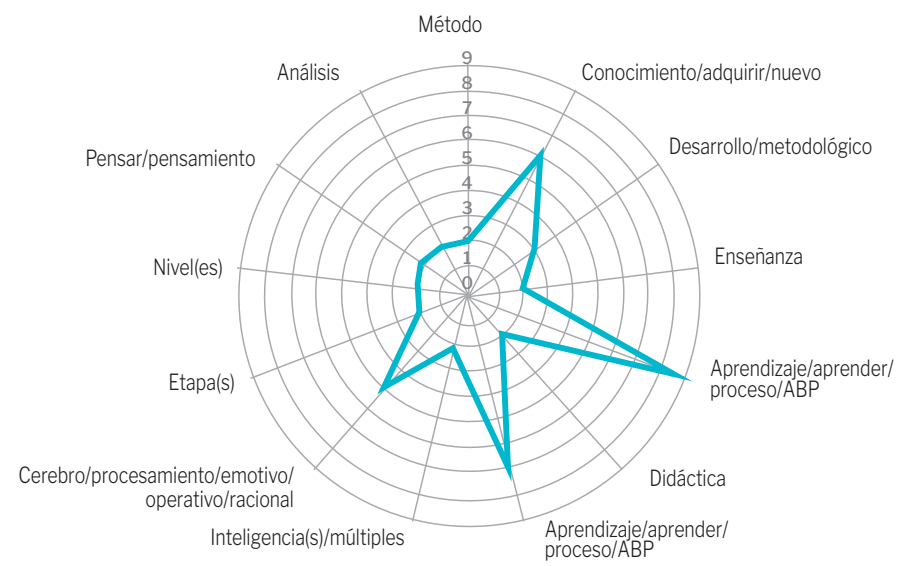

Fuente: Elaboración propia.

Por último, en el término inductor de ambiente, se encuentran asociaciones al lugar, medios y sitios, en cuanto la frecuencia estuvo centrada en la dicción de espacio, al igual que la palabra más importante es la de entorno. En ese sentido, el rango de repetición es de cinco palabras, las cuales hacen referencia a sinónimos de la expresión de mayor frecuencia. 


\section{Análisis de la técnica de tris jerarquizados}

Esta técnica propende a obtener un acercamiento preliminar a los elementos centrales y periféricos del sistema de creencias de la muestra poblacional sobre el rol docente en la modalidad b-learning. De lo anterior, se aplicó a través de 32 fichas, cada una con cierta palabra estímulo derivada de los resultados con mayor frecuencia y aparición en el rango en la técnica de asociaciones libres (tabla 8.1), que tuvo la participación de 15 docentes en razón de la selección determinada en el muestreo por conveniencia (Martín-Crespo Blanco y Salamanca, 2007).

Tabla 8.1. Listado de palabra (fichas) utilizadas en el tris jerarquizado

\begin{tabular}{|l|l|l|l|}
\hline Aprendizaje & Ciencia & Maestro & Tutor \\
\hline TIC & Papel & Características & Docente \\
\hline Tecnología & Desempeño & Experiencia & Trabajo \\
\hline Pedagogía & Actividad & Formación & Proceso \\
\hline Enseñanza & Acompañamiento & Indagar & Cerebro \\
\hline Conocimiento & Guía & Buscador & Relación \\
\hline Herramienta & Facilitador & Curiosidad & Entorno \\
\hline Comunicación & Orientador & Experto & Espacio \\
\hline
\end{tabular}

Nota: Las fichas se digitaron en papeles y se dispusieron de forma individual en un formato de tarjeta. Fuente: Elaboración propia.

\section{Los códigos}

Se codifican las fichas y las respectivas organizaciones efectuadas por los docentes, por ende, se encuentra la fundamentación máxima de quince apariciones en códigos, tales como pedagogía, comunicación, TIC y acompañamiento. Consecuentemente, aparecen códigos con fundamentación con rango de cinco apariciones, toda vez que los códigos con repeticiones inferiores a ese valor, tales como trabajo, buscador, cerebro, experto, espacio, indagar, papel y característica no son tenidas en cuenta para la posterior interpretación a través de co-ocurrencias y consolidación de familias de códigos.

La figura 8.9 muestra la ventana emergente de los códigos en función de la fundamentación que equivale a la frecuencia de aparición en las imágenes tomadas de las jerarquías construidas por la muestra poblacional y, en consecución, los colores asignados para re- 
presentar su rango, en la base (rojo), en el medio (verde), en el ápice inferior (naranja) y en el ápice superior (rojo), el cual es el más importante, teniendo en cuenta la ramificación como estrategia de esquematización.

Figura 8.9. Fundamentación en la codificación abierta.

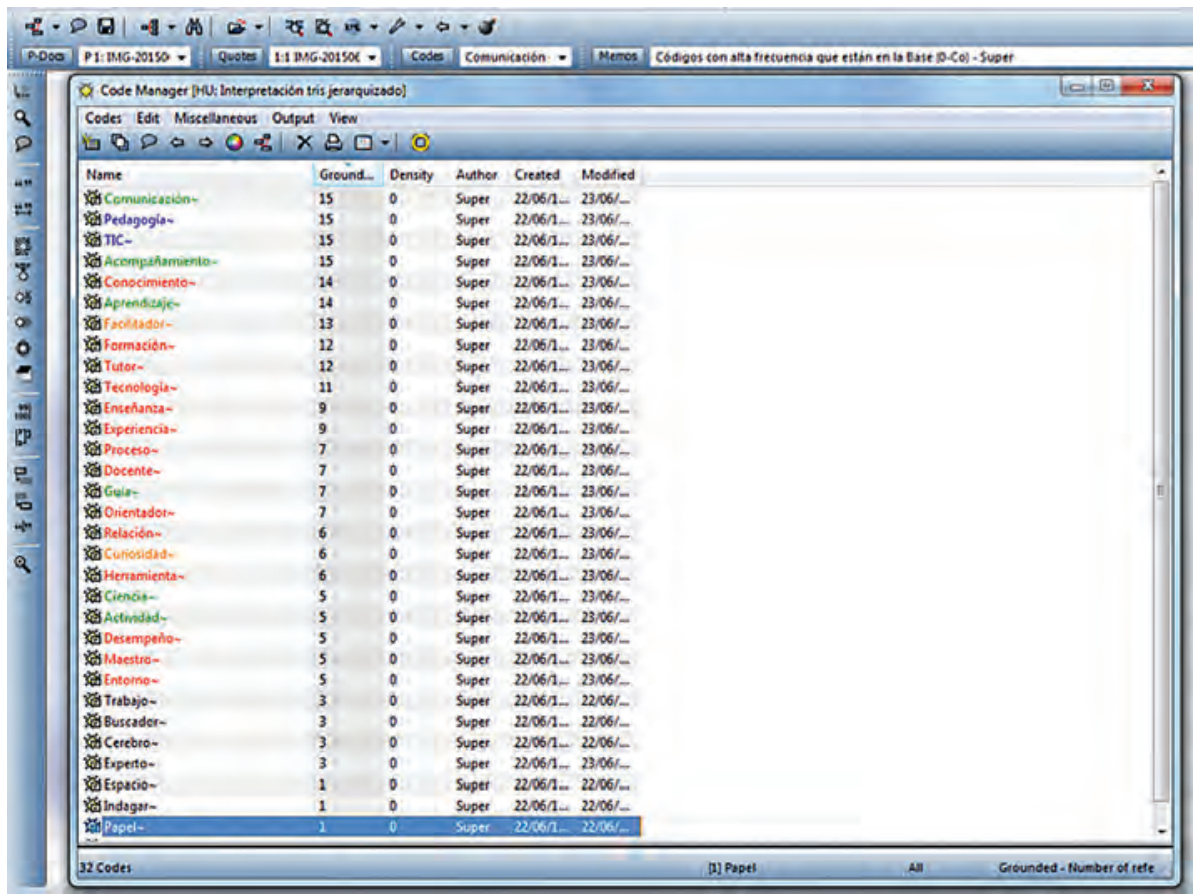

Fuente: Elaboración propia.

\section{Familia de códigos}

Seguido del proceso de codificación, se recurre a la organización de los códigos en familias que agrupen los códigos dependiendo del puesto otorgado entre lo más y menos importante en las jerarquías escalonadas de la técnica de tris jerarquizados. A partir de este ejercicio, se les atribuyen a las familias el nombre figurativo de la posición en la cual se encuentra (base, medio, ápice inferior y ápice superior). Para ello, el software a través de operadores lógicos y la opción query tool encuentra las co-ocurrencias de los códigos por medio de la fundamentación y la densidad. De lo anterior, la mayoría de los códigos residen en la familia de base, la cual se constituye como la parte periférica del contenido representacional; y el ápice superior como la parte rígida o nuclear de la representación social. 
Comunicación, redes, aprendizaje y desarrollo institucional y social

\section{Memorandos}

Strauss y Corbin (2002) definen la codificación axial como "proceso de relacionar las categorías a sus subcategorías, denominado 'axial' porque la codificación ocurre alrededor del eje de una categoría, y enlaza las categorías en cuanto a sus propiedades y dimensiones" (p. 134). Por esta razón, se usan los memorandos que adquieren los siguientes criterios de relación entre familias y códigos adyacentes a otras familias:

- Códigos con alta frecuencia que están en el ápice: este criterio muestra los códigos centrales en la representación social, por tanto, su validez se halla en la correspondencia de frecuencia y grado de importancia dado por los docentes, estando en la cima del contenido representacional.

- Códigos con alta frecuencia que están en la base: caso contrario al presentado en el anterior criterio, hay códigos con frecuencia alta que no aparecen en la cúspide de importancia otorgada por la muestra poblacional; aquellos términos como formación, tutor y tecnología hacen parte de este criterio, que representan la flexibilidad en el contenido representacional.

- Códigos con unanimidad de pertenencia a la familia correspondiente: en algunos casos, la importancia otorgada a cierto código no se dio de manera unánime, por lo cual el puesto por tomar en la jerarquía se otorgó por sumatoria de apariciones en la jerarquía. No obstante, en este criterio, están los códigos cuya unanimidad es absoluta, por tanto, el consenso de importancia es equivalente en toda la muestra poblacional. Estos términos pueden considerarse como primordiales en las definiciones caracterizadoras (Strauss y Corbin, 2002).

- Códigos de baja frecuencia con clasificación de ápice superior o inferior: hay códigos que, en principio, obtuvieron baja frecuencia, pero en la interpretación de la técnica de tris jerarquizados se les otorgó en algunos casos clasificaciones de ápice superior o inferior. Por ejemplo, se encuentran las palabras maestro o relación, que, a pesar de estar en la base de la jerarquía, diversos docentes decidieron colocarlos en lo más alto del esquema.

- Códigos que tienen igualdad en todos los niveles: en el contenido representacional, existen dos términos (curiosidad y guía) que comparten grado de importancia en cada una de las clasificaciones propuestas en la familia de códigos, por lo cual se pueden considerar, desde lo enunciado por Abric (2001), como aquellos términos bisagra para conectar los elementos de cada familia.

- Códigos que están en los diferentes niveles: son los códigos que están presentes en todas las familias, pero no en igualdad de apariciones por cada nivel, es decir, obtienen más aparición en una familia que en otra, lo cual hace que se definan o se cataloguen pertenecientes a un grupo; sin embargo, también pueden fungir como términos bisagra para interconectar cada nivel de la jerarquía. 


\section{Red semántica}

Como última acepción en el análisis de la técnica de tris jerarquizados a través del paradigma de codificación de la teoría fundamentada, se tiene la expresión gráfica del conjunto de elementos ya descritos, por tanto, la puesta en escena de la red semántica construida en el software ATLAS.ti (network) desarrolla en totalidad la relación entre códigos, familias y memorandos para significar las relaciones y jerarquías que adquiere cada componente a través de la fundamentación y el grado de importancia delegado por los docentes (figura 8.10).

Figura 8.10. Red semántica del contenido representacional del rol docente en la modalidad b-learning.

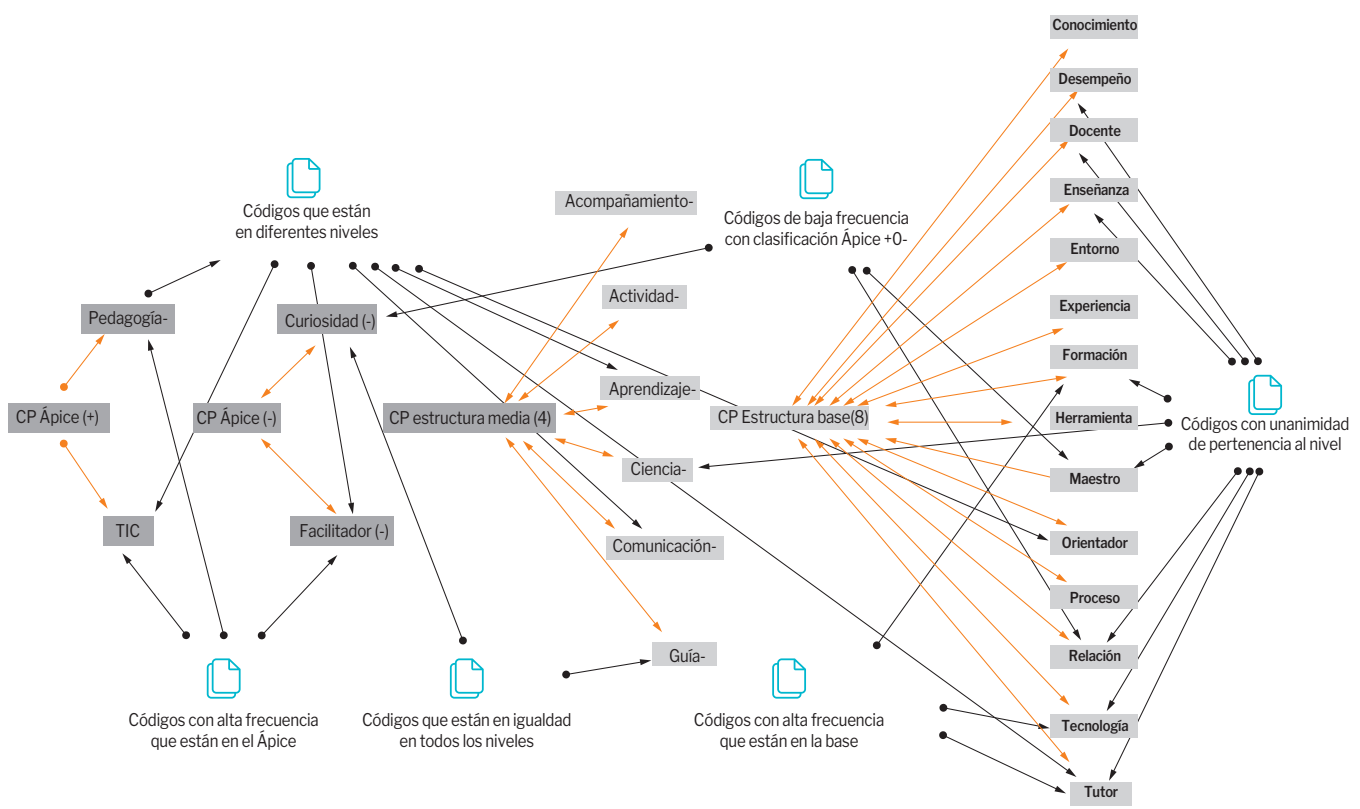

Fuente: Elaboración propia.

\section{Discusión}

Con respecto a la caracterización de las representaciones sociales de los docentes en la Facultad de Estudios a Distancia de la Universidad Militar Nueva Granada y sobre el rol del docente en modalidades b-learning, se encontró en los resultados de las técnicas asociativas (asociaciones libres y tris jerarquizados) que los pensamientos cotidianos en torno a su 
labor se basan en lo pedagógico y en la articulación de las TIC en el campo educativo, reconociendo, en primera instancia, el rol como una acción por desempeñar y, consecuentemente, se identifica la transición del docente tradicional a otro enmarcado en las condiciones de facilitación, orientación, guía y acompañante.

En la categoría de b-learning, se tiene que los procesos de educación se enfocan en el aprendizaje como virtud de estas modalidades, lo cual se profundiza en los tris jerarquizados al mencionar esta palabra en las diferentes jerarquías, tanto en las bases como en los ápices, para describir el conjunto de aspectos pedagógicos, funciones, actividades y entornos que caracterizan la modalidad de aprendizaje semipresencial. De igual manera, las asociaciones del componente pedagógico y de docencia se enmarcan en la enseñanza, toda vez que la referencia de importancia en los resultados adquiridos la catalogan como elemento flexible en el contenido representacional, es decir que hace parte de un atributo característico del rol docente en cuanto las facetas de orientador, maestro y docente, así como también son descritas en la técnica de tris jerarquizados como aspectos inherentes a la base de la estructura representacional.

En otra de las subcategorías establecidas, como el componente psicosocial, en el que intervienen los principios de identidad, reconocimiento, interacción, ambiente, cognición y trabajo flexible, se encuentra preferencia desde las distribuciones de frecuencia de los docentes hacia los componentes cognitivos más que en los sociales. Lo anterior se justifica en la cantidad de aparición de términos inductores seleccionados para caracterizar el rol docente en relación con procesos cognitivos, tales como proceso, curiosidad, actividad, experiencia o conocimiento; en contraste, se tiene que en el grupo de 32 palabras utilizadas en los tris jerarquizados las primeras opciones descartadas fueron las palabras acordes con el papel, el entorno y la relación que contienen aspectos de interacción a través de los principios de identidad, pertenencia y grado de incidencia de los aspectos personales en el quehacer profesional del docente.

\section{Comparación de los resultados con los referentes conceptuales}

Desde las orientaciones y recomendaciones divulgadas por la Unesco (2008) y la OCDE (2009) en relación con el deber ser del docente, cuya transición profesional está condicionada al impacto de las TIC en la educación superior, se tiene que el contenido representacional de los docentes está más afín a esta premura de consolidar el perfil profesional a través de características, tareas y desempeños acordes con la tutoría como modo de actuar y convertirse en guía para la mediación del aprendizaje. Lo anterior se sustenta en la visión prototípica mostrada en los términos inductores presentados en cuanto el perfil y el trabajo flexible, en los cuales no hay consenso de la muestra, pero, a la vez, se retoman como aspec- 
tos que direccionan las acciones profesionales, existiendo el intersticio entre los temas de derechos y responsabilidades (Unesco, 2008) y lo mencionado por este mismo organismo en acopio a lo social, ético y propio del sentido profesional.

En razón de esto, los resultados del contenido representacional volcados a la capacidad del docente para usar las TIC, facilitar el aprendizaje, acompañar a los educandos y fomentar medios, mecanismos y herramientas para la comunicación en la modalidad b-learning, representan la afirmativa a la conjetura expuesta por Eagly (1987) acerca de la apropiación del rol en función exclusiva del trabajo, dejando de lado las implicaciones identitarias y personales que mitigan el conflicto y disenso del rol para con otros actores o agentes de la comunidad educativa.

En esa medida, lo enunciado por Morresi y Donnini (2007) y Méndez, Rivas y Del Toro (2007) en el ámbito latinoamericano sobre la caracterización del rol docente en la tutoría tiene incidencia en el contenido representacional de los docentes de la Universidad Militar Nueva Granada, toda vez que la palabra de tutoría en ambas técnicas asociativas tiene presencia en la fundamentación de los códigos y en la jerarquía a través de la base del diagrama expuesto en la red semántica. Asimismo, lo expuesto por Cataldi et al. (2005); Zambrano, Medina y García (2010) y Dellepiane y Klas (2010) sobre el perfil refuerza el principio de definición del rol a partir del perfil del docente, siendo caracterizado más en las funciones que en el acervo de comportamientos, actitudes y modo de ser del sujeto profesional.

La modalidad b-learning y, en general, el rol del docente para el presente siglo traen consigo cambios profesionales, reconociendo su acción de orientador, facilitador y gestor (Colbert y Castro, 2014). De lo anterior, el contenido representacional de los docentes reafirma la validez de los dos primeros; sin embargo, referentes tales como la gestión, investigación y proyección social no son concebidos como parte de la caracterización del rol, lo cual se refleja en la vaguedad de las cadenas semánticas utilizadas para describir el término investigador, más asociado a la búsqueda y el grado de curiosidad, ratificado en la técnica de tris jerarquizados en la poca aparición de términos, tales como ciencia, indagar, formación y experto, mucho más vinculadas a una de las funciones sustantivas declaradas en la Ley 30/1992 en cuanto a la importancia de la investigación en el quehacer docente.

En el ámbito nacional, también hay correspondencia desde lo dispuesto por la Arboleda y Rama (2013) acerca de la acción del docente facilitador en la modalidad b-learning, por medio de la perfección pedagógica encaminada a la orientación y la motivación de los educandos. Igualmente, para Sabogal (2014), los roles de facilitador, experto en las TIC y consejero hacen parte del deber ser profesional, en cuanto estos elementos se hacen característicos en la parte media y superior de la jerarquía del contenido representacional de 
los docentes. Sin embargo, los aspectos reseñados por el autor como el de líder constructor más afín a la parte social y lo enunciado por el Ministerio de Tecnologías de la Información y Comunicaciones en la alfabetización ciudadana no se encuentran presentes en el conjunto de creencias de la muestra poblacional, toda vez que la parte social aparece aislada de la caracterización del docente, más ajustada a los parámetros de funciones, perfiles y acciones que pueden estar implícitas en el contexto de interacción institucional.

A diferencia del estudio enunciado por Padilla, Páez y Montoya (2008) sobre la creencia de los docentes en el uso de las TIC en la Universidad Militar Nueva Granada que no distinguen las diferencias entre el rol presencial y otro de carácter virtual, la muestra que participó en la investigación exhibe claridad sobre los cambios y las distinciones en el ámbito cognitivo y funcional de las acciones desarrolladas en las modalidades que incorporan las TIC. Sin embargo, las categorías reseñadas por los autores, tales como identidad, actitud y creencias, no son perceptibles en el conjunto de producciones semánticas de las técnicas asociativas. En contraste, los roles caracterizados por González, Padilla y Rincón (2012) en la Universidad Militar Nueva Granada para evaluar al docente en modalidades b-learning presentan afinidad en lo concerniente a la facilitación y la tutoría, no obstante, otros, como el de administrador, diseñador y comunicador, no se identifican en los resultados de este estudio, por lo cual podría haber disyuntiva sobre tales roles y la capacidad de los docentes para apropiarlos o, por el contrario, se conciben como acciones que deben ser desarrolladas por otros profesionales.

\section{Aportes y limitaciones de la investigación}

Uno de los aportes de esta investigación está en la posibilidad de aplicar de forma escalable el conjunto de referentes teóricos y metodológicos acerca del rol docente desde la perspectiva de las representaciones sociales y extrapolarlo con las diferencias y limitaciones contextuales a otras facultades de educación virtual. En ese sentido, la articulación de procesos comprensivos sobre el rol docente permite generar aportes a modelos interpretativos de la política pública educativa en torno al deber ser profesional del docente en estas modalidades, la cual se presenta en plena construcción y pende de reformas más allá de la expuestas en la Ley 30/1992 en el Título primero, capítulo III, y el Decreto 1295/2010 en el apartado de personal docente.

Igualmente, reconocer el contenido representacional del docente permite avanzar hacia el cambio también representacional propuesto por Pozo (2002), con lo cual se tiene que los sujetos adquieren influencia y desarrollo de ideas que pueden ser erróneas sobre determinado objeto social, y esto se refleja en teorías encarnadas que conviven con marcos científicos que intentan remover estos pensamientos basados en la circulación de informa- 
ción en la cotidianidad. Para Moscovici (1996), reconocer las diferencias entre los modelos teóricos y la lógica de los hechos en cuanto a su acontecer en la cotidianidad promueve la actualización de los campos de investigación actuales, por ende, emergen criterios de interpretación y desarrollo de los modelos teóricos que contribuyen a explicar o interpretar la realidad social desde diferentes perspectivas teóricas y metodológicas.

Las representaciones sociales también tienen un papel de regulación en las conductas del sujeto y los grupos, respecto de la afectividad como vínculo de selección de información y organizador de las conductas psicosociales, por ende, los conocimientos desarrollados en ese marco representativo están mediados por las emociones y disertaciones generadas a partir de la comunicación, los intercambios sociales esquemáticos y los procesos cognitivos específicos (Araya, 2002). De la misma manera, para Abric (2001) son una especie de "guía para la acción, orienta las acciones y las relaciones sociales. Es un sistema de pre-descodificación de la realidad puesto que determina un conjunto de anticipaciones y expectativas" (p. 6).

Por otro lado, en cuanto a las limitaciones del estudio, el referente estructural de las representaciones sociales que se plantea para interpretar el contenido de representación del rol docente en la Universidad Militar Nueva Granada no alcanza a desarrollar la hipótesis de centralidad enunciada por Abric (2001). Esta hipótesis de indagación de las centralidades es el último proceso para decantar y establecer si los elementos nucleares o centrales de la representación consecuentemente son los que gobiernan el conjunto de pensamientos cotidianos sobre el quehacer del docente en la modalidad b-learning. Para ello, Abric (2001) describe técnicas como los guiones ambiguos para establecer a través de procesos interrogativos en la muestra la reafirmación de los elementos centrales de las representaciones sociales.

El marco autorrepresentacional hallado en esta instancia de la investigación no permite considerar la incidencia del concepto de self desarrollado por Sarbin (1968) en la teoría del rol, en cuanto no se ha llegado a la instancia de interpretar el marco representacional de los estudiantes y, en general, de otros agentes educativos para establecer relaciones entre las condiciones de emergencia y la dimensión de la representación de la institución sobre el rol del docente en las modalidades b-learning. De esta manera, la siguiente fase de la investigación reconoce la necesidad de este contenido para establecer un modelo teórico tendencial, posible y deseado del objeto social en cuestión.

\section{Resultados en el contexto de la Universidad Militar Nueva Granada}

Los resultados de la investigación enmarcan la posibilidad de procesos formativos, evaluativos y de consideración en el proyecto educativo institucional acerca del rol del docente, más exactamente en lo concerniente a la educación virtual y la planta docente de la Facultad 
de Estudios a Distancia. En razón de esto, el contenido representacional indagado presenta diferentes enfoques en los cuales se puede priorizar intereses y tendencias de profesionalización, como en el caso de la acción facilitadora, la cual ha de profundizarse en referentes pedagógicos y didácticos acordes con esta acción. Igualmente, permite a la Facultad, desde el Centro de Investigaciones, fomentar procesos inclusivos a la investigación educativa y la investigación en educación, en cuanto la distancia de los docentes que interactúan en modalidades b-learning se observa como debilidad en la parte formativa de los profesionales.

La existencia de este mapa de roles compromete una visión reflexiva y prospectiva del quehacer profesional y promueve la perspectiva interpretativa que complemente los elementos tratados en el Acuerdo 4/2004 de la Universidad Militar Nueva Granada concerniente al reglamento del personal docente, mucho más afín a lo enunciado por la Unesco (2008) en torno a la carrera docente, el perfil, los deberes y los derechos y el desarrollo profesional que posee el docente en la institución. La estructura del contenido representacional abre la postura psicosocial respecto de identificar las debilidades y fortalezas en términos comunicativos, identitarios, subjetivos y axiológicos, para reconocerse como agente de cambio, más aún en este tipo de modalidades en las cuales el cambio de protagonismo gira en torno a los estudiantes como principal actor en el proceso educativo.

\section{Conclusiones}

Presentando de forma individual el término inductor del rol a través de la técnica de asociación libre, el reconocimiento de los aspectos de papel y actor adquieren importancia para la muestra poblacional. No obstante, al considerar el conjunto de elementos que caracterizan el contenido representacional (técnica de tris jerarquizado), estos aspectos no son relevantes, teniendo en cuenta que la palabra estímulo papel fue removida del grupo de dieciséis palabras importantes sobre las cuales se ejerce la estratificación final del contenido representacional en todas las organizaciones efectuadas por los sujetos. De esta manera, se puede concluir que en la generalidad de lo enunciado por la muestra poblacional su acción está evocada en relación con funciones, perfiles y desempeños, teniendo en la red semántica la palabra estímulo que deriva de estos componentes.

En el acercamiento a la representación social de los docentes sobre su rol, no se pudo constatar el referente teórico enunciado por Erving Goffman y expuesto por Herrera y Soriano (2004) sobre el compromiso y el disenso del rol, los cuales pueden ser explícitos desde el contraste del marco representacional en otros agentes educativos. Sin embargo, en el acervo de esta teoría en la importancia de la cotidianidad en el rol, se pudieron caracterizar elementos tales como los subroles (facilitador, acompañante, guía, orientador, etc.), las fun- 
ciones manifiestas o latentes que se expresan en el dominio del componente pedagógico y de las TIC para fomentar el aprendizaje desde la experiencia, la curiosidad y la enseñanza como acciones concretas que aporta el docente desde las modalidades b-learning.

\section{Referencias}

Abric, J.-C. (dir.) (2001). Prácticas sociales y representaciones. México: Coyoacán.

Araya Umaña, S. (2002). Las representaciones sociales: ejes teóricos para su discusión. San José, Costa Rica: Facultad Latinoamericana de Ciencias Sociales.

Arboleda Toro, N. y Rama Vitale, C. (eds.) (2013). La educación superior a distancia y virtual en Colombia: nuevas realidades. Bogotá: Virtual Educa.

Cataldi, Z., Figueroa, N., Lage, F., Kraus, G., Britos, P. y García, R. (2005). El rol del profesor en la modalidad de b-learning tutorial. Ponencia presentada en Congreso Internacional Educación Superior y nuevas tendencias, Santa Fe, Argentina, Universidad Nacional del Litoral. Recuperado de http://laboratorios.fi.uba.ar/lsi/rgm/comunicaciones/ CIESyNT-2005-T192.pdf

Cebrián de la Serna, M. y Vain, P. D. (2008). Una mirada acerca del rol docente universitario desde las prácticas de la enseñanza en entornos no presenciales. Pixel-Bit: Revista de Medios y Educación, 32, 117-129.

Colbert, V. y Castro, H. (2014). El nuevo rol del docente para el siglo XXI. Ruta Maestra, 6, 6-9. Dellapiane, P. y Klas, S. (2010). Diseño de contenidos virtuales: experiencia en la materia Turismo Sustentable de la Licenciatura en Turismo y Hotelería de UADE. Ponencia presentada en Congreso Iberoamericano de Educación: Metas 2012, Buenos Aires. Recuperado de http://www.adeepra.org.ar/congresos/Congreso \%20IBEROAMERICANO/ TICEDUCACION/R0793_Dellepiane.pdf

Dorfsman, M. (2012). La profesión docente en contextos de cambio: el docente global en la sociedad de la información. Revista de Educación a Distancia, 6. Recuperado de http:// revistas.um.es/red/article/view/245231

Eagly, A. H. (1987). Sex differences in social behavior: A social-role Interpretation. Hillsdale, $\mathrm{NJ}$ : Eribaum.

González Guerrero, K., Padilla Beltrán, J. E. y Rincón Caballero, D. A. (2012). El docente en contextos b-learning. Bogotá: Universidad Militar Nueva Granada.

Herrera Gómez, M. y Soriano Miras, R. M. (2004). La teoría de la acción social en Erving Goffman. Papers: revista de sociología, 73, 59-79.

Jodelet, D. (2008). El movimiento de retorno al sujeto y el enfoque de las representaciones sociales. Revista electrónica de Ciencias Sociales, 3(5), 32-63. Recuperado de http:// www.scielo.org.mx/scielo.php?pid=S2007-81102008000200002\&script=sci_arttext

Loscertales Abril, F. (1989). Aspectos psicosociales del rol docente. Revista Interuniversita- 
Comunicación, redes, aprendizaje y desarrollo institucional y social

ria del Formación del Profesorado, 6, 541-549. Recuperado de https://dialnet.unirioja. es/servlet/articulo?codigo $=117686$

Martín-Crespo Blanco, M. C. y Salamanca Castro, A. B. (2007). El muestreo en la investigación cualitativa. Nure Investigación, 27, 1-9. Recuperado de http://www.sc.ehu.es/plwlumuj/ ebalECTS/praktikak/muestreo.pdf

Méndez Barceló, A., Rivas Diéguez, A. y Toro Borrego, M. del (2007). Entornos virtuales de enseñanza Aprendizaje. La Habana: Editorial Universitaria.

Ministerio de Educación Nacional (2009). Educación virtual o educación en línea. Recuperado de http://www.mineducacion.gov.co/1621/article-196492.html

Ministerio de Educación Nacional. Decreto 1295 de 2010. Por el cual se reglamenta el registro calificado de que trata la Ley 1188 de 2008 y la oferta y desarrollo de programas académicos de educación superior (20 abril 2010).

Ministerio de Educación Nacional. Ley 30 de 1992. Por el cual se organiza el servicio público de la educación superior (28 diciembre 1992).

Morresi, S. y Donnini, N. (2007). Modalidad de educación semipresencial: relato de una experiencia. Ponencia presentada en el VII Coloquio Internacional sobre Gestión Universitaria en América del Sur, Mar del Plata, Argentina, Universidad Nacional de Mar del Plata. Recuperado de https://repositorio.ufsc.br/bitstream/handle/123456789/82843/ TRABAJODONNINI.pdf?sequence=1

Moscovici, S. (1996). Psicología de las minorías activas. Madrid: Morata.

OCDE (Organización para la Cooperación y el Desarrollo Económicos) (2009). Política de educación y formación. Los docentes son importantes. Atraer, formar y conservar a los docentes eficientes. OCDE. Recuperado de http://www.waece.org/enciclopedia/2/ Los \%20docentes \%20son \%20importantes.pdf

Padilla Beltrán, J. E., Páez Flores, C. M. y Montoya Zapata, R. D. (2008). Creencias de los docentes acerca del uso de las tecnologías de información y comunicación. Revista Educación y Desarrollo Social, 2(2), 45-57. https://doi.org/10.18359/reds.835

Pozo, J. I. (2002). La adquisición de conocimiento científico como un proceso de cambio representacional. Investigações em ensino de ciencias, 7(3), 1-34.

Sabogal Padilla, A. (2014). Innovaciones pedagógicas en b-learning: una experiencia significativa con formación de docentes en el uso y apropiación de TIC. Recuperado de http://reposital.cuaed.unam.mx:8080/jspui/handle/123456789/4072

Salinas, J. (2008). Modelos didácticos en los campus virtuales universitarios: Patrones metodológicos generados por los profesores en procesos de enseñanza-aprendizaje en entornos virtuales. Mallorca: Universitat de les Illes Balears.

Sarbin, T. R. (1968). Notes on the transformation of social identity. En L. M. Roberts, N. S. Greenfield y M. H. Miller (eds.), Comprehensive mental health: The Challenge of evaluation (pp. 97-115). Madison: Universidad de Wisconsin. 
Sautu, R., Boniolo, P., Dalle, P. y Elbert, R. (2005). Manual de metodología: construcción del marco teórico, formulación de los objetivos y elección de la metodología. Buenos Aires: Consejo Latinoamericano de Ciencias Sociales.

Strauss, A. y Corbin, J. (2002). Bases de la investigación cualitativa: técnicas y procedimientos para desarrollar la teoría fundamentada. Medellín, Colombia: Universidad de Antioquia.

Unesco (Organización de las Naciones Unidas para la Educación, la Ciencia y la Cultura y Organización Internacional del Trabajo) (2008). Recomendación conjunta de la OlT y la Unesco relativa a la situación del personal docente (1966) y Recomendación de la Unesco relativa a la condición del personal docente de enseñanza superior (1997). París: OIT. Recuperado de http://unesdoc.unesco.org/images/0016/001604/160495s.pdf

Vaillant, D. (2005). Reformas educativas y rol docente. Protagonismo docente en el cambio educativo, 1, 38-51. Recuperado de http://unesdoc.unesco.org/images/0014/001446/144666s.pdf

Valles, M. S. (2003). Técnicas cualitativas de investigación social reflexión metodológica y práctica profesional. Madrid: Síntesis.

Zambrano, W. R., Medina, V. H. y García, V. M. (2010). Nuevo rol del profesor y del estudiante en la educación virtual. Dialéctica Revista de Educación, 26, 51-61. 